\title{
Study on QoS Gains in Migration from IPv4 to IPv6 Internet
}

\author{
Shailendra S. Tomar, Anil Rawat \\ Computer Division, Raja Ramanna Centre for Advanced Technology, PO CAT, Indore, Madhya Pradesh 452013, India \\ E-mail: tomar@rrcat.gov.in, rawat@rrcat.gov.in \\ Prakash D. Vyavahare \\ Department of Electronics \& Telecommunication Engineering, Shri Govindram Seksaria Institute of Technology and \\ Science, 23 Sir M. Visvesvaraya Marg, Indore, Madhya Pradesh 452003, India \\ E-mail: prakash@sgsits.ac.in, prakash.vyavahare@gmail.com \\ Sanjiv Tokekar \\ Department of Electronics \& Telecommunication Engineering, Institute of Engineering \& Technology, Devi Ahilya \\ Vishwa Vidyalaya, Khandwa Road, Indore, Madhya Pradesh 452017, India \\ E-mail: stokekar@ietdavv.edu.in
}

\begin{abstract}
IPv6 has features, like a) "no header checksum calculation" and b) "no IP packet fragmentation at intermediate routers", which makes it better than IPv4 from router/routing point of view. Existing Internet technology supports both IPv6 and IPv4 protocols for transport of packets and hence dual addressed machines are widely present. Maximizing QoS in IPv6 networks, as compared to IPv4 networks, for sites having dual addresses is an active area of research. Results of our study on QoS gains in networks connected to IPv6 Internet as compared to IPv4 Internet for a network of about 2500 nodes are presented here. The technique used to estimate QoS gains in the migration from IPv4 to IPv6 is also presented. The test-bed data of one month with 25000 most visited websites was analyzed. The results show that an alternate IPv6 channel exists for a large number of major global websites and substantial QoS gains in terms of reduced access times - averaging up to $35 \%$ for some websites - can be expected by intelligent per site IP address selection for dual stack machines.
\end{abstract}

Index Terms - IPv4 to IPv6 migration, QoS gains, dual stack machines, per site IP address selection.

\section{INTRODUCTION}

Migration of IPv4 [1] networks to IPv6 [2] networks over the Internet have been extremely slow because of many factors. The most well-known factors are a) Security concerns in IPv6, b) Lack of proper training of most of the network administrators on IPv6, c) Unpreparedness of Internet Service Providers (ISPs) to provide reliable IPv6 services, d) No strong reason to shift to IPv6 for those who already have required IPv4 addresses, e) Lack of measurement technique to measure Quality of Service (QoS) gains in migrating from IPv4 to IPv6. Therefore, it is estimated that the complete migration from IPv4 to IPv6 networks will take a substantial amount of time, which may range from anywhere between 5 years to 10 years. This has prompted researchers to carry out investigations on performance in such a heterogeneous Internet.

In present day Internet scenario, almost all small and midsized IPv4 networks are mostly connected to the Internet using the IPv4 to IPv4 Network Address Translation (NAT) technique. IPv6 is used only when a large number of addresses are required or the Internet Service Provider (ISP) cannot provide IPv4 addresses. The world over, over the last couple of years, ISPs are gearing up for providing IPv6 addresses. This has resulted in a large number of networks having IPv6 testbed setups aimed at gaining more experience of working on IPv6 networks. It is a well-known fact that IPv6 will not appeal to users unless some substantial performance gain over IPv4 is seen by the users or unless there is no other mode of connectivity available to connect to the Internet.

We have carried out a detailed study of IPv6 protocol and current Internet configuration trends, with an eye on factors which can really provide performance gains over existing IPv4 only networks. Number of studies have been carried out in the past by a large number of researchers, but mostly their studies are focused on the technical comparison of IPv4 and IPv6 protocols. In this paper, we are focusing on the features of IPv6 and the present Internet configuration trends which are expected to provide performance gains over IPv4. We have setup a test-bed, which is connected to the Internet for testing them. We have also developed some scripts using commonly used network tools, which can give us the desired output for comparing the two technologies with respect to QoS enhancement for website accesses. We have based our study on an organization which mostly has web users. Real network access log files have been scanned for finding out the accessed websites over the 
network for a substantial period of time. While performing the tests for IPv4 path, the IPv4 network is connected to the Internet using the NATing technique, while for testing of IPv6 path, routed network has been considered.

In this paper, we are presenting our results of a study on QoS gains in IPv6 networks as compared to IPv4 networks for accessing sites having dual (both IPv4 and IPv6) addresses and are presenting a technique to estimate the overall effective QoS gains for users in the migration from IPv4 to IPv6 in an organization. We have setup an experimental test-bed for a network connected to the Internet, both by using IPv6 and IPv4 forms of connectivity, and the results for a network of 2500 nodes are presented in this paper.

It can be inferred from the results that an alternate IPv6 channel exists for a large number of major global websites and substantial QoS gains ( averaging up to $35 \%$ for some websites ) can be expected by intelligent per site IP address selection for dual stack machines. We expect that our results will encourage users across the world to adopt IPv6 in a large way and as a result contribute towards early adoption of IPv6.

The paper is organized as follows. Section I gives a brief introduction to the subject area of the research work. In section II, the study of literature related to the paper is discussed, followed by section III which covers the hypothesis of this research work. Section IV briefly describes the research methodology used for the study. Section V describes the Methods, Tools, and Techniques used in the research work. In section VI, the results section, we publish the major findings of the research. Section VII presents result analysis and inferences drawn. VIII is the future works related section which describes areas where further research work is possible and section IX contains conclusive remarks.

\section{STUDY OF LITERATURE}

IPv4 and IPv6 protocols have different architectures and hence are not interoperable. Various studies have been carried out related to the performance analysis [3][4][5][6][7] of the IPv4 and IPv6 protocols. All these studies highlight design level differences between the two. Providing QoS [8][9] guarantees over the Internet have been a subject of research ever since its inception. With invariable delays in transmission, processing, and propagation of packets across the Internet, users experience nondeterministic service quality in best effort IP networks.

For a best possible experience of a service in a network QoS requirement for any service is defined by four parameters a) minimum bandwidth required b) maximum permissible latency c) maximum permissible jitters and d) maximum allowed packet losses. For website accesses, QoS is affected by access times. Hence, QoS gain can be calculated by determining the percentage reduction in access time as compared to the earlier access time. On the Internet, guaranteed QoS is provided using differentiated QoS model [10] or Integrated QoS model [11]. In our study, we are considering only best effort services without any special QoS priority settings on intermediate routers.

Although IPv6 has a number of advantages over IPv4, the most well-known is the availability of a large number of addresses. The availability of IPv4 to IPv4 Network Address Translation (NAT) [12][13][14], as an alternate solution to the IPv4 address shortage problem, has undervalued this advantage. This has been a major factor behind the extremely slow migration of networks from IPv4 to IPv6.

IPv6 protocol has a number of key features, out of which a) "no header checksum calculation" and b) "no IP packet fragmentation at intermediate routers", can enhance the QoS at the end user. Some networks may also have different paths for IPv4 and IPv6 services and hence may have different hop counts and Maximum Transmission Units (MTUs) for these paths. A path with a smaller hop count and larger MTU value in IPv6 network may also enhance the QoS for the end user. Added to these, with only a few networks having access to IPv6 part of the Internet, it is also possible that users accessing the IPv6 part of the Internet may experience lesser congestion and hence get better QoS. With more and more Distributed Denial of Service DDoS attacks [15] aimed at IPv4 networks it gives IPv6 network users the advantage of expecting better QoS in best effort networks. Another factor that may result in skewed performance of IPv4 over IPv6 networks is the difference in configurations at the intermediate nodes. For example, enabling of fast forwarding (Application Specific Integrated Circuit (ASIC) based routing) for IPv4 packets and slow forwarding (memory based routing) for IPv6 packets or vice versa, in intermediate nodes. It is also possible that a different set of resources - in addition to the ones assigned to IPv4 networks - are assigned by organizations for IPv6 networks.

All the above-mentioned factors make it difficult to ascertain a deterministic QoS for the end user in best effort IP network. Depending on these factors, the QoS of various services using IPv4 and IPv6 transport modes of connectivity may vary significantly on per connection basis. Hence, to make the best use of available resources, it is imperative to use both the IPv6 and IPv4 transport layer, with selective routing based on the estimated QoS gains of the user for a service over that path at that point in time. Already existing set of QoS-aware routing protocols [16][17][18][19] can be enhanced to support selective routing based on IPv6 network QoS profile gains.

The selection of the path with the best QoS value at a particular moment from the available set of IPv4 and IPv6 paths, prior to the start of the session, is bound to enhance the user level experience in best effort networks. There are a number of IPv4 to IPv6 transition methods to enable users to start using IPv6 addressing. Study on the performance of these methods has been made in [20][21][22]. In [23] congestion aware routing in IPv6 has been discussed. This paper proposes and presents techniques to use commonly available network 
performance measurement tools. This will enable us to differentiate between the best performing IPv4 and IPv6 paths, based on access times at the start of a session. We have used the technique mentioned below and gathered results for a network of 2500 nodes.

\section{HYPOTHESIS}

In today's Internet, both IPv4 and IPv6 forms of connectivity exists for various services. For services available on the Internet, using the IPv6 form of connectivity, following factors may lead to enhanced QoS to users, as compared to the IPv4 form of connectivity:

a) No header checksum calculation for IPv6 packets.

b) No packet fragmentation at intermediate routers in IPv6 packets.

c) The possibility of different and higher value MTU configuration along the IPv6 path.

d) The possibility of different and lower hop count because of a different path for IPv6 packets.

e) IPv6 part of the Internet is accessed by a fewer number of users. Hence lesser congestion can be expected.

f) The possibility of enabling fast forwarding feature for IPv6 packets, and slow forwarding for IPv4 packets, in intermediate nodes.

g) The possibility of assignment of an additional set of resources for IPv6 connectivity.

h) The absence of NATing delays on the IPv6 path.

i) The absence of complex packet filtering rules at the routers along the IPv6 path.

j) A lesser number of hackers in IPv6, due to its lesser popularity, leading to lesser Denial of Service (DoS) attacks.

Thus, our hypothesis, states that "In present day Internet, depending on various router and node level configurations, web services offered over IPv6 networks may provide better QoS to users as compared to IPv4 networks in some cases".

Our investigations in this paper are on best effort services only, i.e services without any QoS-related priority configurations on the intermediate routers on the Internet. We have considered QoS gains of such services caused due to reduced access times. Values of rest of the parameters like the packet loss, bandwidth and jitter are assumed to be similar in IPv4 and IPv6 networks.

\section{RESEARCH METHODOLOGY}

The research methodology followed in this paper for estimating the user-level QoS gains in migration from IPv4 to IPv6 is depicted in Fig. 1.

In the first step, we collect the historic web access logs covering all websites accessed by the organizational users. The Internet is a huge resource, hosting more than 1 billion websites. All websites are not relevant to an organization since organizations have specific objectives and its constituent users use the Internet primarily to meet those objectives. Hence, we have to first find the websites which are relevant to the organization. To find out these websites the easiest approach is to scan the web access logs of the organization. In our case, we scanned the squid proxy access logs of our organization to filter out all websites accessed over a period of one month by the users. A period of one month was chosen to account for all users who may be on leave for extended durations up to two to three weeks.

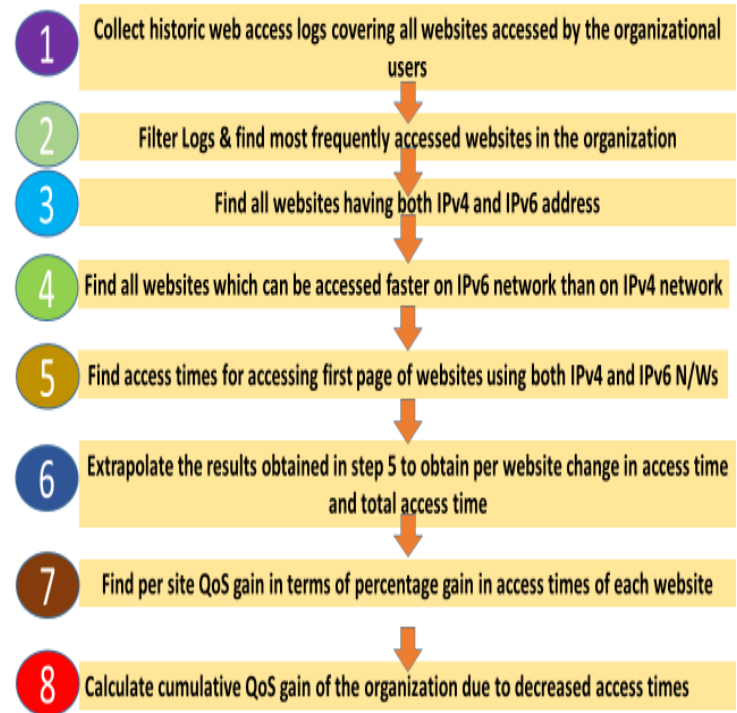

Fig.1. Research methodology for estimation of QoS gains in migration from IPv4 to IPv6

The next step is to filter out the visited websites and find the websites which are of higher relevance to the users of the organization. This is done since a large number of websites will be accessed by the users of the organization, but to measure QoS gains to users of the organization as a whole, only top few websites will contribute significantly towards the QoS gains. Here it can be safely assumed that most websites accessed will be common to users of the organization. We can determine the number of websites to consider for further analysis, by multiplying the number of serious Internet users in the organization by the average number of websites accessed by the users of the organization. The average number of different websites accessed by users in the organization can be safely taken as 100 . This number can be increased for more accurate results.

In the next step we find out all websites having both IPv4 and IPv6 addresses, from among the websites selected in step 2. This is required since we have to compare the QoS gains for migration from IPv4 to IPv6. This makes sense only for a website which has IPv6 address along with an IPv4 address.

In the next step we find out all websites which can be accessed faster on the IPv6 network than on an IPv4 network. Since we are considering here that all websites which are either slower to access in the IPv6 network can alternately be accessed in IPv4 network. Therefore the user need not shift to an IPv6 mode of connectivity for 
that website. This will give us all websites which have better access times using IPv6 mode of connectivity and hence will contribute towards the calculation of QoS gains in migration from IPv4 to IPv6.

Step 5 is to find access time values for accessing only the first page of each website, for both IPv4 and IPv6 networks, for sites contained in the list generated in step 3 . This parameter will allow us to find the difference in access times between IPv4 and IPv6 networks for each website. For website accesses, QoS is inversely proportional to the access times. Hence, the QoS gain for a particular website can be ascertained from the change in access time value of the website.

Step 6 is to find the contribution of change in access time of each website towards the calculation of the QoS gain for the entire organization. Since our goal is to develop a technique for estimating the QoS gain for the organization as a whole, and the QoS gain contribution of each website is directly proportional to the amount of downloads performed on the websites, hence we extrapolate the change in access times for the homepage to find the change in access times due to the total downloads from each website.

Step 7 is to find the QoS gain of each website, in percentage with respect to the access times using the IPv4 network.

In the last step i.e step 8, we estimate the QoS gains as a holistic indicator for the organization. The QoS gain calculated in this manner takes into account only access time parameter, assuming everything else is the same for both IPv4 and IPv6 networks.

\section{Methods, Tools, AND TeChNiQues}

For calculating QoS gains of an organization in migration from IPv4 to IPv6, we need to first find the QoS gain contribution of each website accessed by the users of the organization. We need to consider only those websites which are relevant to the organization and for which both IPv6 and IPv4 addresses exist. For finding the QoS gain due to one website, we follow the following steps:

a) We first find the access time of the homepage of the website using IPv4 and IPv6 networks separately. Then, we subtract the access times of the website for an IPv4 network from the access times of the same website for an IPv6 network, to get the difference in access times caused by shifting from IPv4 to IPv6 network.

b) We also find the number of bytes downloaded while accessing the homepage of a website. Next, the change in access times for accessing the homepage with IPv4 and IPv6 network is extrapolated to find the total change in access times for downloading all the pages/bytes from that website. The total number of pages/bytes downloaded for a particular website has been obtained from the proxy server logs that are collected for sites accessed in the organization.
To find the per site QoS gain of a website in percentage, we divide the difference in access time of the website by the access time required in an IPv4 network and multiply that by 100 . Thus, the following formula is used for finding the QoS Gain for a Website in Percentage (QGWP):

$$
\mathrm{QGWP}=\frac{(E A T v 4-E A T v 6) * 100}{E A T v 4}
$$

Here EATv4 is the Extrapolated access time for a website in IPv4 network and EATv6 is the Extrapolated access time for the website in IPv6 network

For finding the overall QoS gain of the entire organization, we add all the extrapolated changes in access times of all the websites and the extrapolated access time obtained using the IPv4 network. To find the QoS gain of the entire organization in percentage, we divide the added up extrapolated changes with the added up extrapolated access time calculated using the IPv4 network and multiply it by 100 . Thus, the following formula is used for finding the QoS Gain for the entire Organization in Percentage (QGOP):

$$
\mathrm{QGOP}=\frac{\sum_{i=1}^{i=n}(E A T i, v 4-E A T i, v 6) * 100}{\sum_{i=1}^{i=n} E A T i, v 4}
$$

Here EATi,v4 is the Extrapolated access time for $i^{\text {th }}$ website in IPv4 network and EATi,v6 is the Extrapolated access time for the website in IPv6 network and value of $i$ ranges from 1 to $n$ where $n$ is the maximum number of websites for which IPv6 access time is lesser than IPv4 access time.

For the collection of data related to the analysis, we enabled log module in the squid [16] proxy server and created a log file for recording websites accessed from our organization on a daily basis. Then, by using log aggregation technique, day wise historic web access log files have been aggregated to create a single log file for the entire month. The aggregation has been done by using "bash" scripts in Linux, mainly based on the "cat" command line tool. The aggregated access log file has been further parsed to obtain top 25000 websites accessed by the users of test organization within a period of one month time. This has been done to cover all types of user accesses from our test network. All websites obtained after parsing, are further probed for the presence of both "A" and "AAAA" name server records from the "google" public DNS server with IP 8.8.8.8. DIG tool has been used to probe the DNS server.

QoS gain has been estimated using both PING and CURL tools. For finding websites with better response time using IPv6 connectivity as compared to using IPv4 connectivity, CURL tool has been used. The tool has been used to obtain response times of the website both by using the IPv4 network and IPv6 network. PING tool has been used to obtain response times of the server both using the IPv4 network and an IPv6 network. For measuring HOP COUNTS, the output of PING command has been parsed for Time To Live (TTL) value. For 
filtering of data, "awk" and "grep", utilities have been used. PHP scripts have been written for analysis of the data. PHPGRAPHLIB library of PHP has been used for plotting the results.

\section{A. Test Setup}

Figure 2 depicts a test-bed setup created to test the hypothesis. IPv4 connectivity has been simulated using NAT to depict the true situation of IPv4 networks connected to the Internet. IPv6 connectivity has been achieved using a pure IPv6 only router connected to the National Knowledge Network (NKN) of India.

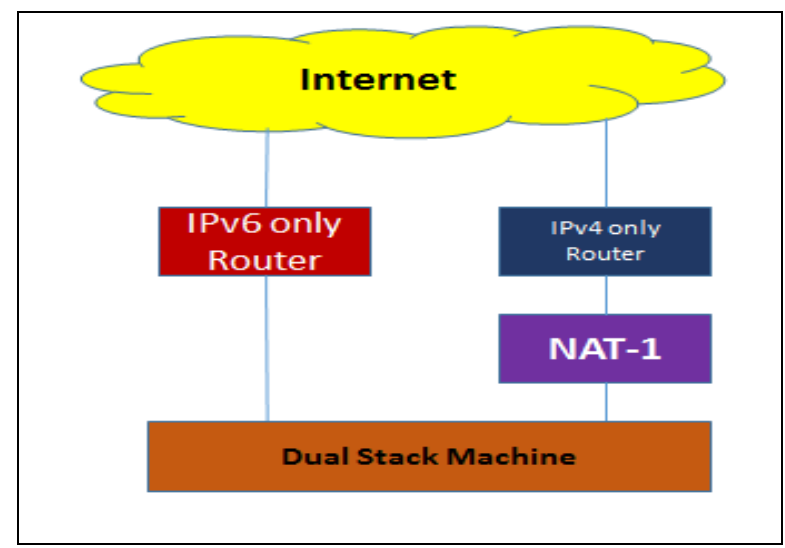

Fig.2. Test-bed setup for simulating IPv4 connectivity using NAT and IPv6 using pure routed network

\section{B. Data Filtering and Analysis scripts}

The following set of scripts are written to perform various data collection and analysis tasks:

1) Script to find top 25000 websites relevant to the organization:

We have used the free opensource script "Squid TopSite Report Generator (STSRG)" which is written in Perl and is available on the URL http://davehope.co.uk/projects/ perl-squid-reporting/. This script allows us to find the top 25000 websites by download size, in our test organization using the consolidated squid access logs for a period of one month.

2) Script to find all websites with both A and AAAA type address records:

The list of top 25000 websites by download size is scanned. Using the DIG tool and the public name server we find the AAAA and A records respectively by the following commands:

$$
\begin{aligned}
& \text { - } \quad \text { dig @ 8.8.8.8 \$SITENAME -t AAAA } \\
& \text { - } \quad \text { dig @ 8.8.8.8 \$SITENAME -t A }
\end{aligned}
$$

Then by using "awk" and "grep" commands and the "bash" shell script utilities the output of DIG command is filtered to obtain IP addresses.

\section{3) Scripts for RTT collection and analysis}

The list of all websites with both IPv4 and IPv6 addresses is scanned. Using the PING tool the RTT values for both IPv6 and IPv4 addresses are determined respectively by the following commands:

$$
\begin{array}{ll}
\text { - } & \text { ping6 -M do -c } 3 \text {-s \$PKT_SIZE \$IPv6 } \\
\text { - } & \text { ping -M do -c } 3 \text {-s \$PKT_SIZE \$IPv4 }
\end{array}
$$

PHP scripting language has been used to develop a program for analysis of the output of the abovementioned commands. The difference in RTT times for IPv4 and IPv6 addresses is calculated for each of the sites. Sites having IPv6 RTT values lesser than IPv4 RTT values are categorized into different groups of reductions between $0-10 \%, 10-20 \%, 20-30 \%, 30-40 \%, 40-50 \%, 50-$ $60 \%, 60-70 \%, 70-80 \%, 80-90 \%, 90-100 \%$. Finally, the plot of the percentage of websites against percentage reduction in the RTT values is generated.

\section{4) Scripts for Hop Count collection and analysis:}

The list of all websites with both IPv4 and IPv6 addresses is scanned. Using the PING tool the TTL values for both IPv6 and IPv4 addresses are determined respectively by the following commands:

$$
\begin{aligned}
& \text { - } \quad \text { ping6 -M do -c } 3 \text {-s \$PKT_SIZE \$IPv6 } \\
& \text { - } \quad \text { ping -M do -c } 3 \text {-s \$PKT_SIZE \$IPv4 }
\end{aligned}
$$

PHP script has been written to analyze the output generated by the above-mentioned commands. String processing commands have been used for this purpose. TTL values for both IPv4 and IPv6 addresses of the same site are compared and the difference is recorded.

5) Scripts for web sites response collection and analysis:

Following commands have been used for collection of access times of the first page of each of the site having both IPv6 and IPv4 addresses.

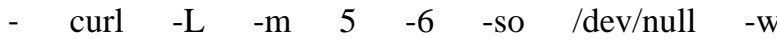

$$
\begin{aligned}
& ' \%\{\text { time_total }\} \quad \%\{\text { size_download }\} \backslash n ' \\
& \text { HTTP://\$SITE }
\end{aligned}
$$

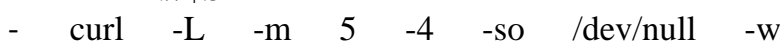

$$
\begin{aligned}
& \text { ' } \%\{\text { time_total }\} \quad \%\{\text { size_download }\} \backslash n \text { ' } \\
& \text { HTTP://\$SITE }
\end{aligned}
$$

PHP script has been written to analyze the output of the above commands and extrapolate the output to count total reduction in access times and the total projected access time using IPv4 mode of connectivity. Sites with IPv6 access time values lesser than IPv4 access time values are categorized into 10 different groups of reductions in steps of i.e 0-10;10-20 etc.. Finally, the plot of the percentage of websites against a percentage reduction in the access times values is generated using the 
PHPGRAPHLIB library. The percentage QoS gain for the entire organization (QGOP) is also calculated using the (2).

\section{RESULTS}

Tools and techniques as discussed in the previous section have been applied on a test network of 2500 nodes. The results of our study of web related accesses have been published here. In our test network, all the users use squid proxy for accessing the web. Squid proxy logs collected for a period of one month have been used as test data set to find the web sites access related parameters in our study. The probe tests using Ping and Curl tools were conducted multiple times at different times of the day and on different days. Almost similar results were achieved every time.

Various results relevant in determining the quality of service gain in the organization in selectively using the IPv6 network and IPv4 networks are as follows:

1) Number of websites relevant to our organization $=$ $4,63,628$

2) Number of websites with both IPv4 and IPv6 website addresses out of top 25000 websites accessed in our test organization:

a. Sites which responded to both v6 and v4 Ping requests $=3234$

b. Sites which responded to both v6 and v4 Curl requests $=3438$

3) Percentage of websites with both IPv4 and IPv6 addresses out of top 25000 websites accessed in our test organization $=13.752 \%$

4) Number of websites with IPv6 RTT lesser than IPv4 RTT $=912$

5) Percentage of websites with IPv6 RTT lesser than IPv4 RTT from among the websites accessible using both IP6 and IPv4 address $=28.2 \%$

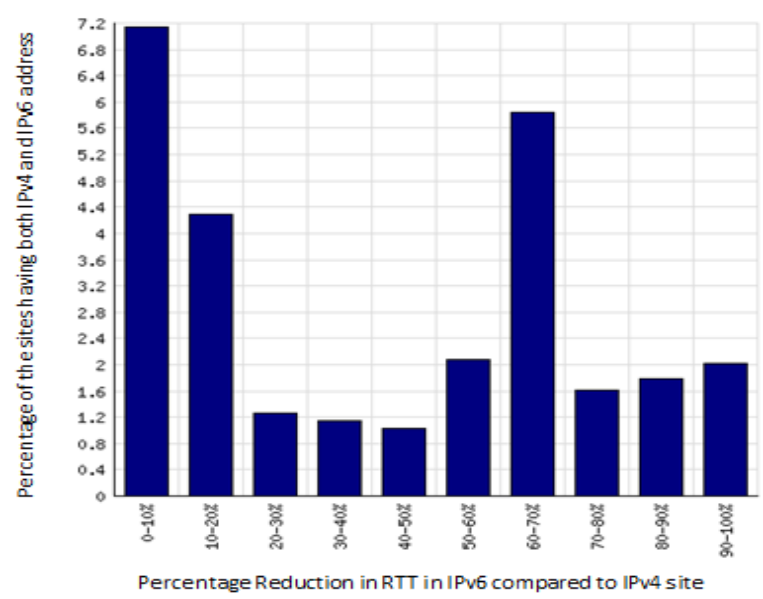

Fig.3. Plot of the percentage of websites having both IPv4 and IPv6 addresses with percentage reduction in RTT in IPv6 compared to IPv4
6) Number of websites with IPv6 web access time lesser than IPv4 web access time $=1454$

7) Percentage of websites with IPv6 RTT lesser than IPv4 RTT from among the websites accessible using both IP6 and IPv4 address $=42.2 \%$

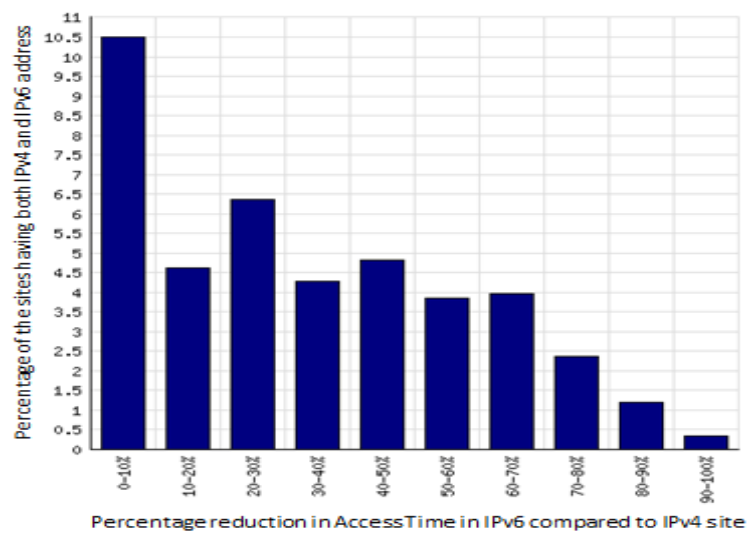

Fig.4. Plot of percentage of websites with reduced IPv6 access times as compared to IPv4 against percentage reduction in the access times

8) Number of websites with hop count lesser in IPv6 than that for IPv4 = 21

9) Estimated QoS gains for the test organization calculated using (2) as mentioned in section V, is approximately $34.36 \%$ in test case under consideration.

\section{INFERENCES}

The inferences drawn from the results of the data analysis using the proposed tools and techniques after normalizing the various values to be compared, in percentage form, are as follows:

1) In the test organization, only $0.05 \%(4,63,628$ / $1,038,274,900)$ of the total number of websites in the world are accessed. This is as expected since large number of web sites are blocked as a matter of policy in the organization.

2) Out of the top 25000 accessed in our test organization, $13.72 \%$ websites have valid IPv6 addresses. This is also as expected since IPv6 adoption is happening at a very slow rate globally.

3) Out of all dual addressed websites $28.2 \%$ websites have lesser IPv6 RTT than IPv4 RTT values. This is largely due to the various factors as discussed in our hypothesis in section III.

4) $42.2 \%$ websites, out of all dual addressed websites, have lesser IPv6 web access time than IPv4 web access time. Note that the difference in the number of websites having a lesser percentage of reduced IPv6 RTT as compared to IPv4 RTT may be due to the policy of filtering of ICMP packets for some sites.

5) Estimated QoS gain for our test organization in migration from IPv4 to IPv6 is $34.36 \%$. The QoS gain is calculated based on the estimated reduction in access times of the IPv6 websites with a better 
communication channel.

6) Overall, $5.78 \%$ of top 25000 websites accessed in our test organization have QoS gains of up to $34.36 \%$ when accessed using the IPv6 network as compared to the IPv4 network.

Therefore, it can be clearly inferred from the results that there is a justified reason for organizations to start using the dual IP addressing scheme as QoS gains in migration from IPv4 to IPv6 are substantial and cannot be ignored.

\section{FUTURE WORK}

The QoS estimation technique presented in this paper reveals the advantage of the migration from IPv4 to IPv6 network for the organization as a whole. As the migration from IPv4 to IPv6 is a slow process hence both types of connectivity are required in the organization. Dual stack machines, which can connect to the Internet both by using IPv4 and IPv6 connectivity modes, decide to choose from either IPv4 or IPv6 mode of connectivity by following algorithms as defined in RFC 6724. RFC 6724 defines algorithms for the default source and destination address selection in case dual stack services are available on a machine. A site wide address selection approach can provide better access experience to the user. Hence, our future course of work will be to generate QoS gain profiles on per website basis for different points of time. QoS gain profiles thus generated can be referred to by dual stack machines to decide which IP address to use for best QoS at that point in time. It is expected to increase the Quality of Experience (QoE) for services accessed from QoS gain profile enabled networks.

\section{CONCLUSION}

In today's Internet, for best effort services, three major factors that affect the QoS are a) the protocol selection (IPv6/IPv4), b) Internet configurations and c) the congestion on the path of communication. The selection of protocol is one of the important decisions to make in dual stacked machines in present Internet which supports both IPv4 and IPv6 protocols for transport of data.

IPv6 protocol has been designed for better routing of packets over the Internet. Presently, due to various reasons, access delays in both IPv4 and IPv6 networks vary to a great extent as explained in detail in this paper. IPv6 channel of connectivity exists for a large number of major global websites. Internet configuration for the IPv6 part of the Internet is still not fully optimized and the process is still going on. As has been described and elaborated in this paper, access times of various websites are different on different channels of Internet. All organizations should consider this fact and try to utilize the benefits of the alternate IPv6 channel that exist on the Internet, by proper channel selection.

The technique presented in this paper for estimating QoS gains for the organization in migration from IPv4 to
IPv6 will help an organization in finding their overall QoS gain to users due to the migration. The results achieved in our test network are encouraging. We hereby conclude that QoS gains of the order of $30-40 \%$ can be straightaway expected for about $6 \%$ websites which are accessed by organizations. QoS gains will increase with time since IPv6 networks are still being fine tuned all across the world.

\section{REFERENCES}

[1] Cerf V. and Kahn R.E., "A protocol for packet network inter-communication", IEEE Transactions on Communications,Vol. 22, No.5, pp 637-648, May 1978.

[2] Deering S. and Hinden R., "Internet Protocol Version 6 (IPv6) Specification," RFC 2460, Dec. 1998, http://www.ietf.org/rfc/rfc2460.txt.

[3] Y. Sookun and V. Bassoo, "Performance analysis of IPv4/IPv6 transition techniques," 2016 IEEE International Conference on Emerging Technologies and Innovative Business Practices for the Transformation of Societies (EmergiTech), Balaclava, 2016, pp. 188-193.

[4] J. Hyun, J. Li, H. Kim, J. H. Yoo and J. W. K. Hong, "IPv4 and IPv6 performance comparison in IPv6 LTE network," Network Operations and Management Symposium (APNOMS), 2015 17th Asia-Pacific, Busan, 2015, pp. 145-150.

[5] N. Chuangchunsong, S. Kamolphiwong, T. Kamolphiwong, R. Elz and P. Pongpaibool, "Performance evaluation of IPv4/IPv6 transition mechanisms: IPv4-inIPv6 tunneling techniques," The International Conference on Information Networking 2014 (ICOIN2014), Phuket, 2014, pp. 238-243.

[6] M. Ahmed, A. Litchfield, S. Ahmed, A. Mahmood, Md. E. Hossain, "VoIP Performance Analysis over IPv4 and IPv6," International Journal of Computer Network and Information Security, vol.6, no.11, pp.43-48, October 2014.

[7] S. Shivani and Monalisa "Comparison analysis of various transition mechanisms from IPv4 TO IPv6" International Journal Of Engineering And Computer Science vol. 2 no. 62013.

[8] S. Deng, H. Wu, D. Hu and J. Leon Zhao, "Service Selection for Composition with QoS Correlations," in IEEE Transactions on Services Computing, vol. 9, no. 2 pp. 291-303, March-April 12016.

[9] L. Li, M. Rong and G. Zhang, "An Internet of things QoS estimate approach based on multi-dimension QoS," Computer Science \& Education (ICCSE), 2014 9th International Conference on, Vancouver, BC, 2014, pp. 998-1002.

[10] Blake S., D. Black, M. Carlson, E. Davies, Z. Wang, and W.Weiss, "An Architecture for Differentiated Services, “ RFC 2475,December 1998.

[11] Braden R., D. Clark, and S.Shenker, "Integrated Services in the Internet Architecture: an Overview," RFC 1633, June 1994.

[12] R. Ghafouri, A. Ashrafi and B. V. Vahdat, "Security consideration of migration to IPv6 with NAT (Network Address Translation) methods," 2015 23rd Iranian Conference on Electrical Engineering, Tehran, 2015, pp. 746-749.

[13] V. J. D. Barayuga and W. E. S. Yu, "Packet Level TCP Performance of NAT44, NAT64 and IPv6 Using Iperf in the Context of IPv6 Migration," 2015 5th International Conference on IT Convergence and Security (ICITCS), 
Kuala Lumpur, 2015, pp. 1-3.

[14] P. Srisuresh and M. Holdrege, "IP Network Address Translator (NAT) Terminology and Considerations," RFC 2663, Aug, 1999.

[15] Muhammad Aamir, Muhammad Arif, "Study and Performance Evaluation on Recent DDoS Trends of Attack \& Defense", IJITCS, vol.5, no.8, pp.54-65, 2013. DOI: 10.5815/ijitcs.2013.08.06

[16] Baygi M.J., Ramli A.R.B., Ali B.M.,Mashohor S., Q-Net, A novel QoS Aware Routing Algorithm for future Data Networks. World Academy of Science, Engineering and Technology 712010.

[17] M. Kodialam; T. V. Lakshman. Minimum Interference Routing with Applications to MPLS Traffic Engineering, In Proceedings of the IEEE Infocom, 2000.

[18] S. Suri; M. Waldvogel; P. R. Warkhede. Profile-based routing: A new framework for mpls traffic engineering, In Quality of Future Internet Services, Lecture Notes in Computer Science 2156, September 2001.

[19] W. Szeto; R. Boutaba; Y. Iraqi. Dynamic Online Routing Algorithm for MPLS Traffic Engineering, International Conference in Networking, 2002.

[20] Adira Quintero, Francisco Sans and Eric Gamess,"Performance Evaluation of IPv4/IPv6 Transition Mechanisms", International Journal of Computer Network and Information Security(IJCNIS), Vol.8, No.2, pp.1-14, 2016.DOI: 10.5815/ijcnis.2016.02.01

[21] S. Aravind and G. Padmavathi, "Migration to Ipv6 from IPV4 by dual stack and tunneling techniques," 2015 International Conference on Smart Technologies and Management for Computing, Communication, Controls, Energy and Materials (ICSTM), Chennai, 2015, pp. 107111.

[22] Erik Chromy and Tomas Behul,"Measurement Based Admission Control Methods in IP Networks", IJITCS, vol.5, no.10, pp.1-8, 2013. DOI: 10.5815/ijitcs.2013.10.01

[23] Matej Kultan and Martin Medvecký,"Congestion Aware Multipath Routing: Aggregation Network Applicability and IPv6 Implementation", IJITCS, vol.7, no.11, pp.9-16, 2015. DOI: $10.5815 /$ ijitcs.2015.11.02

\section{Authors' Profiles}

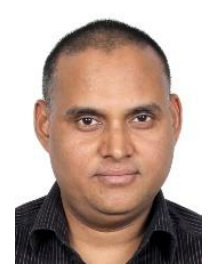

Shailendra Singh Tomar received an M.E degree in Computer Science from Shri Govindram Seksaria Institute of Technology and Science, Indore, India in 2003. He is currently working in Computer Divison of Raja Ramanna, Centre for Advanced Technology, Indore, India and also pursuing his Ph.D. work. His reaserach interests include IPv6 Performance, Security and QoS analysis.

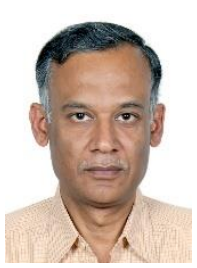

Anil Rawat received an M.E degree in Computer Engineering and a Ph.D. in Electronics and Communications from Devi Ahilya Vishwavidyala, Indore, in the years 1991 and 2008 respectively. He is currently leading the Computer Division in Raja Ramanna, Centre for Advanced Technology, Indore, India. His research interests include Adhoc Networks, Network Security and Big Data analysis.

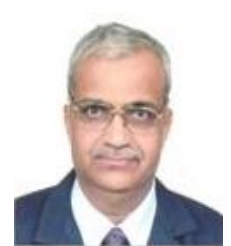

Prakash D.Vyavahare received his M.Tech and Ph.D. degrees in Electronics Engineering from IIT Mumbai in 1976 and 1995 respectively. He worked at Tata Institute of Fundamental Research, Mumbai as communication engineer during 1976 to 1982. He was associate of ICTP (UNESCO organization), Trieste, Italy during 1998 to 2005. He is currently working as Professor in Department of Electronics and Telecommunications Engineering, Shri Govindram Seksaria Institute of Technology and Science, Indore, India. His research interests include channel coding, channel modeling, cross layer design issues and secure communication.

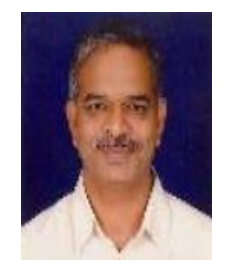

Sanjiv Tokekar received M.E and Ph.D degrees in Electronics Engineering from Devi Ahilya Vishwa Vidyalaya (DAVV), Indore in 1985 and 1996 respectively. He is currently working as Professor, Director and Head of the Department of Electronics and Telecommunications Engineering at Institute of Engineering and Technology, DAVV, Indore. His areas of interest include Computer Networking, Computer Architecture, Performance evaluation of computer systems and microcontrollers.

How to cite this paper: Shailendra S. Tomar, Anil Rawat, Prakash D. Vyavahare, Sanjiv Tokekar,"Study on QoS Gains in Migration from IPv4 to IPv6 Internet", International Journal of Information Technology and Computer Science(IJITCS), Vol.9, No.5, pp.1-8, 2017. DOI: 10.5815/ijitcs.2017.05.01 